\title{
Uterine cervical carcinoma treated with chemoradiotherapy: impact of three-month MRI follow-up on clinical management and outcome
}

\author{
Helena Sundström ${ }^{1,2}$, Lennart Blomqvist ${ }^{1,2}$, Kristina Hellman ${ }^{3,4}$ \\ 'Department of Molecular Medicine and Surgery, Karolinska Institutet, Stockholm 17176, Sweden. \\ ${ }^{2}$ Department of Imaging and Physiology, Karolinska University Hospital, Stockholm 17176, Sweden \\ ${ }^{3}$ Department of Women's and Children's Health, Karolinska Institutet, Stockholm 17176, Sweden. \\ ${ }^{4}$ Department of Gynaecologic Cancer, Theme Cancer, Karolinska University Hospital, Stockholm 17176, Sweden.
}

Correspondence to: Dr. Helena Sundström, Department of Molecular Medicine and Surgery, Karolinska Institutet, Stockholm 17176, Sweden. E-mail: helena.sundstrom@ki.se

\begin{abstract}
How to cite this article: Sundström H, Blomqvist L, Hellman K. Uterine cervical carcinoma treated with chemoradiotherapy: impact of three-month MRI follow-up on clinical management and outcome. J Cancer Metastasis Treat 2021;7:65.

https://dx.doi.org/10.20517/2394-4722.2021.139
\end{abstract}

Received: 22 Jun 2021 First Decision: 12 Jul 2021 Revised: 21 Jul 2021 Accepted: 2 Aug 2021 Published: 9 Nov 2021

Academic Editors: Ting-Chang Chang, Lucio Miele Copy Editor: Yue-Yue Zhang Production Editor: Yue-Yue Zhang

\begin{abstract}
Aim: The aim of this study was to evaluate the impact of MRI performed three months after treatment on further follow-up interventions and outcome in patients with uterine cervical carcinoma treated with definitive chemoradiotherapy.
\end{abstract}

Methods: Sixty consecutive women diagnosed with uterine cervical cancer FIGO 2009 stage IB1-IVA during 20112012 treated with definitive chemoradiotherapy/radiotherapy with curative intent at the Department of Gynaecological Oncology at Karolinska University Hospital were retrospectively included. A review of MRI reports and medical records with focus on follow-up interventions associated to imaging was performed.

Results: On follow-up MRI three months post treatment, 29/60 women had complete remission (mrCR), 24/60 women had partial remission ( $\mathrm{mrPR}$ ) and 7/60 had progressive disease (mrPD). In patients with mrCR, no additional procedures were performed. The group with mrPR had 27 additional MRIs, 3 PET/CT examinations and 9 biopsy procedures, none leading to diagnosis of residual tumour. Locoregional control rate was $96 \%$ after 6.5 months (median). No patient had cervical relapse only; $2 / 53$ had cervical relapse in combination with non-regional lymph nodes and distant relapse. There was no statistically significant difference in overall survival between

The Author(s) 2021. Open Access This article is licensed under a Creative Commons Attribution 4.0 International License (https://creativecommons.org/licenses/by/4.0/), which permits unrestricted use, sharing, adaptation, distribution and reproduction in any medium or format, for any purpose, even commercially, as long as you give appropriate credit to the original author(s) and the source, provide a link to the Creative Commons license, and indicate if changes were made. 
patients with $\operatorname{mrCR}$ and $\operatorname{mrPR}(\mathrm{HR}=2.2, P=0.21)$.

Conclusion: Patients with residual changes on MRI at three months post treatment have a low risk for locoregional recurrence. If this is not recognised, follow-up MRI results in unnecessary additional procedures with low impact on treatment outcome. Further studies are needed regarding the most appropriate imaging modality and timing of post-treatment evaluation.

Keywords: Uterine cervical carcinoma, chemoradiotherapy, locoregional control, MRI, post-radiation changes, relapse, residual tumour, natural course

\section{INTRODUCTION}

Uterine cervical carcinoma (CC) is the fourth most common malignancy among women worldwide and the fourth leading cause of cancer-related death in this group. Around $85 \%$ occur in less developed regions ${ }^{[1]}$. CC commonly affects young women, with a peak incidence in Sweden at the age of 30-50 and about twothirds of the cases occur before 60 years of age. In Sweden, 533 new cases were reported to the National Cancer Registry in $2019^{[2]}$.

Forty per cent of patients have locally advanced disease at diagnosis (FIGO 2009 stage 1B2-IVA), and standard treatment since early 2000 is a combination of external beam radiation therapy, brachytherapy and chemotherapy [definitive chemoradiotherapy (CRT)]. If there is parametrial invasion and/or pathological lymph nodes on MRI, patients with clinically staged FIGO 2009 stage IB1 sometimes also receive definitive $\mathrm{CRT}^{[3-8]}$. In 2018, the FIGO staging system was revised to include lymph node status on imaging or pathology ${ }^{[0]}$.

In locally advanced CC, at least one third of the patients later develop recurrence or progressive disease after definitive CRT. Most recurrences occur within 2-3 years after the primary treatment ${ }^{[10]}$. About $20 \%$ of the recurrences are locoregional, while the majority consist of non-regional lymph node metastases and/or distant metastases ${ }^{[11]}$. Local recurrences can be treated by salvage hysterectomy in selected cases ${ }^{[12]}$, while regional and distant metastases are mainly treated with chemotherapy or radiotherapy (depending on the extent and localisation).

Magnetic resonance imaging (MRI) has become the imaging modality of choice for pre-treatment T-staging of locally advanced stages greater than FIGO 2009 IB1 to delineate tumour, estimating tumour size and location, invasion of the parametria, infiltration of the pelvic side walls and adjacent organs ${ }^{[13-15]}$. In the Stockholm-Gotland region, patients referred to Karolinska University Hospital since 2003 for CC all have MRI of the pelvis as a routine part of the pre-treatment workup.

MRI is also used for follow-up after treatment with CRT to evaluate treatment response ${ }^{[16]}$. The treatment effect on the tissues makes it difficult to differentiate between changes caused by radiotherapy from presence of residual tumour, especially if the MRI is performed shorter than three months after completed treatment ${ }^{[17]}$.

In cases of complete response, no further MRI is usually performed. However, when there is only a partial response on MRI at three months, the most appropriate management is a more difficult decision as the natural course and potential spontaneous regression of the lesion is not known. In dubious cases, a new MRI may be performed no sooner than after an additional eight weeks ${ }^{[18]}$. 
Current surveillance routines lack evidence regarding the most effective workup to diagnose recurrence that would determine long-term outcome. The best timing for follow-up with MRI is not known, nor is the most appropriate management depending on the result of MRI.

The aim of this study was therefore to retrospectively investigate the results of MRI performed at three months after CRT and evaluate its impact on further follow-up interventions and outcome regarding relapse and survival.

\section{METHODS}

\section{Study population}

Patients were identified in the Swedish Quality Registry of Gynaecological Cancer.

The inclusion criteria were all women diagnosed with primary CC FIGO 2009 stage IB1-IVA treated with radiotherapy/CRT with curative intent at the Department of Gynaecological Oncology at Karolinska University Hospital in Stockholm between 2011 and 2012. Exclusion criteria were metastatic disease before first follow-up MRI after treatment, history or diagnosis of another malignancy during follow-up or contraindications to MRI.

A review of medical records and MRI reports regarding date of and age at diagnosis, smoking, histology, FIGO stage, lymph node metastases, treatment, date of therapy (start and finish), date for evaluation of treatment, date of MRI examinations, MRI reports, localisation and time of relapse, last date of follow-up, status at follow-up and date of death was performed. The patients were followed in retrospect until 15 December 2017.

Of 173 women diagnosed with biopsy-verified CC, 113 were excluded for reasons shown in Figure 1. The final study group consisted of 60 patients.

All patients underwent clinical examination under general anaesthesia and staging according to FIGO $2009^{[8]}$. Cystoscopy and rectoscopy were only performed in cases of clinical suspicion of tumour involvement of bladder or rectum. Patients performed an MRI a median 26 days (range 8-104 days) before treatment and 3 months (range 2-4 months) after the treatment was finished. The result of the pretreatment MRI affected treatment decision, but not the clinical FIGO staging. Before treatment, all patients also performed a CT scan of the abdomen and thorax.

\section{MRI}

All MRI examinations three months after finished treatment were performed at the same department. Seven MRI examinations were obtained in other institutions, two pre-treatment and five post-treatment (median 45 months, range 29-69 months), but reviewed at Karolinska University Hospital.

MRI at Karolinska was performed using a 1.5 T system (Siemens AERA, Erlangen, Germany or Philips Achieva, Philips Medicial Systems Best, The Netherlands) using a body array coil. To reduce degradation of imaging quality due to bowel motion, an anti-peristaltic agent (glucagon $1 \mathrm{mg}$, Novo Nordisk, Buscopan 20 mg, Sanofi Aventis) was administered intramuscularly.

For assessment of locoregional disease, high-resolution sagittal and transverse T2-weighted turbo spin-echo sequences of the lesser pelvis were performed followed by transverse T1-weighted turbo spin-echo sequence to evaluate lymph nodes and bone marrow. T2-weighted oblique turbo spin-echo images parallel and 


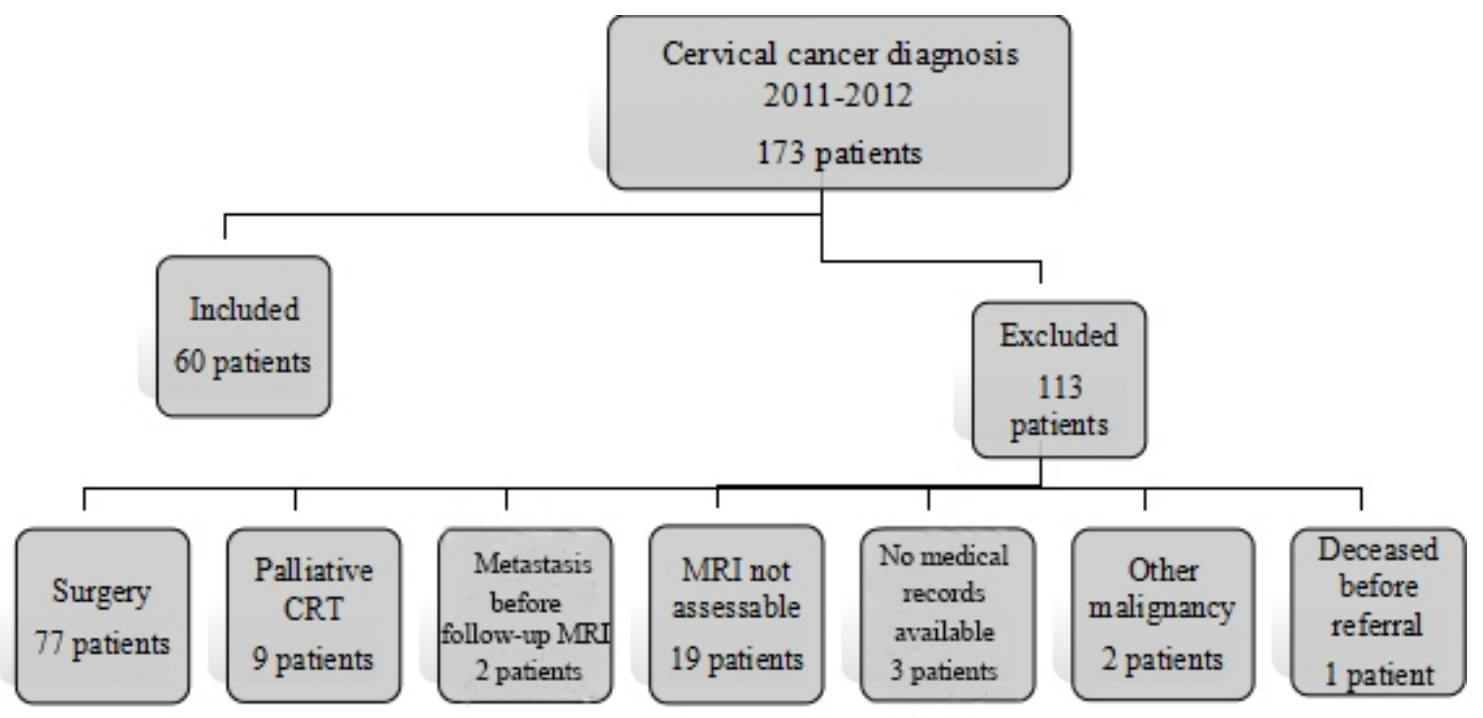

Figure 1. Reasons for exclusion.

perpendicular to the cervix were then obtained. The upper abdomen was examined using respiratory triggered T2-weighted turbo spin-echo technique and T1-weighted gradient echo images with fat saturation before and after intravenous administration of standard dose $(0.2 \mathrm{~mL} / \mathrm{kg}$ body weight $)$ i.v. gadolinium chelate contrast agent (gadoterate meglumine, DOTAREM, Guerbet France), from the top of the liver to the promontory. Finally, the lesser pelvis was examined with a T1-weighted fat saturated post-contrast sequence.

Two patients (3\%) did not receive i.v. contrast during their pre- or post-treatment MRI, one because of impaired renal function and one who refused i.v. contrast. From May 2012, diffusion-weighted gradientecho EPI sequences with $b$ values of 50 and $800 \mathrm{~s} / \mathrm{mm}^{2}$, respectively, from the top of the liver to below the symphysis were added to the protocol ${ }^{[17]}$. In total, $24 / 60(40 \%)$ and $34 / 60(57 \%)$ had DWI included in their MRI protocol before treatment and three months after the end of CRT, respectively.

Follow-up (FU) MRI within 2-4 months after the end of CRT was classified as three months (FU MR3m). FU MRI within 5-7 months after the end of CRT was classified as six months (FU MR6m).

All MR examinations were prospectively interpreted as part of the clinical routine by two radiologists, at least one of them with experience in gynaecological imaging. Tumour response on MRI at three months was evaluated by comparing with baseline pre-treatment MRI.

\section{Treatment}

Patients were treated with EBRT (3D conformal external beam radiotherapy, intensity-modulated radiotherapy or volumetric arc therapy) and intracavitary brachytherapy (ICBT). Radiotherapy was completed in a median of 44 days (range 37-65 days). The standard dosage was 45 Gy to the whole pelvis and 50 Gy to the tumour volume as SIB (simultaneous integrated boost) in 25 fractions.

ICBT was given with pulsed-dose-rate (PDR), 2-3 times, except for some patients who received high-doserate (HDR), 3-4 times. The PDR was given with 8 Gy/fraction and HDR with 6 Gy/fraction with dose 
prescription to ICRU (International Commission of Radiation Unit) point A and orthogonal X-rays for treatment planning. The ICBT was given once a week during the last weeks of EBRT. Patients $(n=5)$ who could not receive ICBT had an EBRT boost to the central tumour with 2 Gy daily up to a total dose of 66-76 Gy.

Patients with para-aortic lymph node metastases on the pre-treatment MRI received an extended field radiation up to the level of T12-L1 (45 Gy in 25 fractions). An additional sequential boost was given to the lymph node metastases with a daily fraction size of 2 Gy to a total dose of 55-64 Gy (depending on localisation and size of the lymph node metastasis).

All patients were planned to receive concurrent weekly intravenous chemotherapy (cisplatin, $40 \mathrm{mg} / \mathrm{m}^{2}$ ), six cycles. Due to comorbidities, 8/60 (13\%) patients could not receive concurrent cisplatin.

\section{Follow-up}

According to the 2008 Swedish regional treatment guidelines, MRI was performed three months after the completion of CRT for treatment evaluation. In cases of complete response, no additional MRI was performed. All patients had clinical examinations at the Department of Gynaecologic Oncology at Karolinska University Hospital every third month the first year and every fourth month the second year. Later clinical controls were performed in outpatient care every sixth month for another three years ${ }^{[19]}$.

Recurrence was defined as a biopsy- or cytology-verified new tumour discovered after FU MR3m in a patient with no evidence of disease at MR3m.

\section{Retrospective evaluation of MRI reports}

According to the retrospective evaluation of radiology reports of FU MR3m, locoregional treatment response was defined as:

. Complete remission (mrCR): No described residual tumour within the irradiated area.

- Partial remission (mrPR): Any statement in the report indicating remaining signal intensity changes in the cervical area where residual tumour could not be excluded, with or without a recommendation to perform an additional follow-up MRI.

- Stable disease (mrSD): Tumour extent or size similar to that before treatment.

- Progressive disease (mrPD): Any description of increase in extent or size of tumour or lymph nodes or the development of new lesions.

\section{Statistics}

Before analysis, some of the data were processed. The patients were divided into four groups: FIGO 2009 stage IB1-IB2, IIA-IIB, IIIA-IIIB and IVA, respectively. Histology was divided into squamous and nonsquamous including adenocarcinoma and adenosquamous. Tumour size on pre-treatment MRI was divided into 2-4 and $>4 \mathrm{~cm}$. Smoking habits were divided into two groups: never smokers and present/previously smokers. Associations between categorical variables were tested using the Pearson's Chi-square test or the Fisher's exact test when appropriate. 
The follow-up time for the whole group was calculated from the date of FU MR3m to the date of death or date of last follow-up. Time to first recurrence for patients with $\mathrm{mrCR}$ and $\mathrm{mrPR}$ was calculated from the date of FU MR3m. Time to death was calculated from the date of FU MR3m.

Overall survival was illustrated using Kaplan-Meier curves and the hazard ratios were estimated with Cox $\mathrm{PH}$ regression analysis. Univariable and multivariable/multiple analysis were calculated using Cox $\mathrm{PH}$ regression. $P$-values $<0.05$ were considered statistically significant.

\section{RESULTS}

Clinical and histopathological characteristics and status at last follow-up of the 60 patients in the study are described in Tables 1 and 2. FU MR3m was assessed as complete response (mrCR) in 29 patients (48\%), partial response (mrPR) in $24(40 \%)$ and progressive disease (mrPD) in 7 patients (12\%). No patients had mrSD.

\section{Management of patients with mrPR}

In 14/24 patients assessed as mrPR, there were measurements of the suspected residual tumour ranging 0.5$5 \mathrm{~cm}$. In the remaining 10 patients, there were no measurements of residual tumour in the radiology reports but descriptions such as areas of incomplete fibrosis, remaining inhomogeneous signal changes or increased contrast enhancement.

No additional procedures were performed in the group with mrCR at FU MR3m. In the group with mrPR on FU MR3m, the following additional procedures were performed because of suspected residual tumour: 27 MRIs, 3 FDG-PET/CT examinations and 9 biopsy procedures [Figure 2].

On average, each patient with mrPR had almost two additional procedures. None of these additional procedures led to diagnosis of residual disease or recurrence. The FDG-PET/CTs showed complete metabolic response in all cases. In none of the biopsies taken due to suspected residual cervical tumour on FU MR3m were viable tumour cells found.

Twelve of the 24 patients in $\operatorname{mrPR}(50 \%)$ were finally assessed as CR, although there was almost no change in the MRI appearance of their suspicious residual cervical tumour on further follow-up examinations, after a median time of nine months (range 6-14 months). The locoregional control rate was $96 \%$ after a median time of seven months (range 3-14 months) in the group with mrPR and for the entire study population $92 \%$ after a median time of three months (range 2-14 months) after FU MR3m.

There was no significant difference between those who had MRI with DWI protocol or not in the distribution of mrCR and mrPR at FU MR3m (data not shown).

\section{Recurrence and survival}

Thirteen out of 53 patients (25\%) relapsed during time of follow-up. Table 3 shows the locations of relapses in one or multiple organ systems for the mrCR and mrPR groups. In total, 5/53 patients (9\%) relapsed within the previously irradiated area. None of the patients had recurrent disease in the cervix or regional lymph nodes only. Two patients with mrPR had local recurrence in the cervix in combination with distant metastases (non-regional lymph nodes and peritoneal carcinomatosis) after 12 and 56 months, respectively. Patients with mrCR had distant recurrences alone in $80 \%$, compared to $38 \%$ in the $\mathrm{mrPR}$ group. The recurrences were mostly discovered due to symptoms that generated medical contact and not on routine check-ups. Median time to recurrence in mrCR and mrPR from FU MR3m was 15 months (range 3-33 
Table 1. Clinical and histopathological characteristics

\begin{tabular}{|c|c|c|c|c|c|}
\hline Variables & Whole group $(n=60)$ & $\operatorname{mrCR}(n=29)$ & $\operatorname{mrPR}(n=24)$ & $\operatorname{mrPD}(n=7)$ & P-value \\
\hline \multicolumn{6}{|l|}{ Age, years } \\
\hline Median (range) & $56(25-85)$ & $54(27-75)$ & $59(35-85)$ & $54(25-81)$ & NS \\
\hline \multicolumn{6}{|l|}{ Smoker* ${ }^{\star} n(\%)$} \\
\hline Yes & $14(23)$ & $7(24)$ & $4(17)$ & $3(43)$ & NS \\
\hline No & $24(40)$ & $13(45)$ & $9(4)$ & $2(29)$ & \\
\hline Previously & $22(37)$ & $9(31)$ & $11(46)$ & $2(29)$ & \\
\hline \multicolumn{6}{|l|}{ Histology, n (\%) } \\
\hline Squamous & $49(82)$ & $24(83)$ & $19(79)$ & $6(86)$ & NS \\
\hline Non-squamous & $11(18)$ & $5(17)$ & $5(21)$ & $1(14)$ & \\
\hline \multicolumn{6}{|l|}{ FIGO stage, $n(\%)$} \\
\hline IB1/IB2 & $12(20)$ & $10(34)$ & $2(83)$ & $0(0)$ & 0.041 \\
\hline$\| \mathrm{A} / \mathrm{IIB}$ & $32(53)$ & $15(52)$ & $14(58)$ & $3(43)$ & \\
\hline IIIA/IIIB & $15(25)$ & $3(10)$ & $8(33)$ & $4(57)$ & \\
\hline IVA & $1(2)$ & $1(3)$ & $0(0)$ & $0(0)$ & \\
\hline \multicolumn{6}{|l|}{ Primary tumour size ${ }^{\star \star}, n(\%)$} \\
\hline $2-4 \mathrm{~cm}$ & $20(33)$ & $14(48)$ & $6(25)$ & $0(0)$ & NS \\
\hline$>4 \mathrm{~cm}$ & $34(57)$ & $14(48)$ & $14(58)$ & $6(86)$ & \\
\hline Not measured & $6(10)$ & $1(3)$ & $4(17)$ & $1(14)$ & \\
\hline \multicolumn{6}{|l|}{ Parametrial invasion ${ }^{\star \star}, n(\%)$} \\
\hline Yes & $52(87)$ & $22(76)$ & $24(100)$ & $6(86)$ & NS \\
\hline No & $7(12)$ & $6(21)$ & $0(0)$ & $1(14)$ & \\
\hline Uncertain & $1(2)$ & $1(3)$ & $0(0)$ & $0(0)$ & \\
\hline \multicolumn{6}{|c|}{ Lymph node metastasis ${ }^{\star \star}, n(\%)$} \\
\hline Yes (pelvic) & $25(42)$ & $10(34)$ & $11(46)$ & $4(57)$ & NS \\
\hline Yes (pelvic and para-aortic) & $2(3)$ & $0(0)$ & $2(8)$ & $0(0)$ & \\
\hline No & $32(53)$ & $19(66)$ & $10(42)$ & $3(43)$ & \\
\hline Uncertain & $1(2)$ & $0(0)$ & $1(4)$ & $0(0)$ & \\
\hline \multicolumn{6}{|l|}{ Chemoradiation, n (\%) } \\
\hline Yes & $52(87)$ & $28(97)$ & $19(79)$ & $5(71)$ & NS \\
\hline $\mathrm{No}^{\star \star \star}$ & $8(13)$ & $1(3)$ & $5(21)$ & $2(29)$ & \\
\hline \multicolumn{6}{|l|}{ Relapse ${ }^{\star \star \star \star}, n(\%)$} \\
\hline Yes & $13(25)$ & $5(17)$ & $8(33)$ & $N A^{\star \star \star \star \star}$ & NS \\
\hline
\end{tabular}

${ }^{\star}$ At time of diagnosis; ${ }^{* \star}$ on initial MRI before treatment; ${ }^{\star \star \star}$ radiation only; ${ }^{\star \star \star \star}$ local, regional and/or distant $(n=53) ;{ }^{* \star \star \star \star}$ NA: not applicable.

Table 2. Results at last follow-up

\begin{tabular}{lllll}
\hline Status at last follow-up, $\boldsymbol{n}(\%)$ & Whole group $(\boldsymbol{n}=\mathbf{6 0})$ & $\mathbf{m r C R}(\boldsymbol{n}=\mathbf{2 9})$ & $\mathbf{m r P R}(\boldsymbol{n}=\mathbf{2 4})$ & $\mathbf{m r P D}(\boldsymbol{n}=\mathbf{7})$ \\
\hline Follow-up time ${ }^{\star}$ median (range) & $58(1-76)$ & $59(7-75)$ & $58(17-76)$ & $9(1-40)$ \\
Alive without CC & $41(68)$ & $25(86)$ & $16(67)$ & $0(0)$ \\
Palliative treatment for CC & $1(2)$ & $0(0)$ & $1(4)$ & $0(0)$ \\
Dead because of CC & $16(27)$ & $4(14)$ & $6(25)$ & $6(86)$ \\
Dead because of other causes & $2(3)$ & $0(0)$ & $1(4)$ & $1(14)$ \\
\hline
\end{tabular}

*From follow-up MRI three months post treatment, in months.

months) and 22 months (range 3-56 months), respectively. 
Table 3. Location of recurrence in patients with $\mathrm{mrCR}$ and $\mathrm{mrPR}$ on FU MR3m $(n=13)$

\begin{tabular}{lll}
\hline Location of recurrence $(\boldsymbol{n})$ & $\mathbf{m r C R}(\boldsymbol{n})$ & $\mathbf{m r P R}(\boldsymbol{n})$ \\
\hline Cervix alone (0) & 0 & 0 \\
Pelvic lymph nodes alone (0) & 0 & 0 \\
Paraaortic lymph nodes alone (1) & 0 & 1 \\
Distant alone (7) & 4 & 3 \\
Cervix and pelvic lymph nodes (0) & 0 & 0 \\
Cervix, pelvic lymph nodes and distant (0) & 0 & 0 \\
Cervix and distant (2) & 0 & 2 \\
Pelvic lymph nodes and distant (3) & 1 & 2 \\
\hline
\end{tabular}

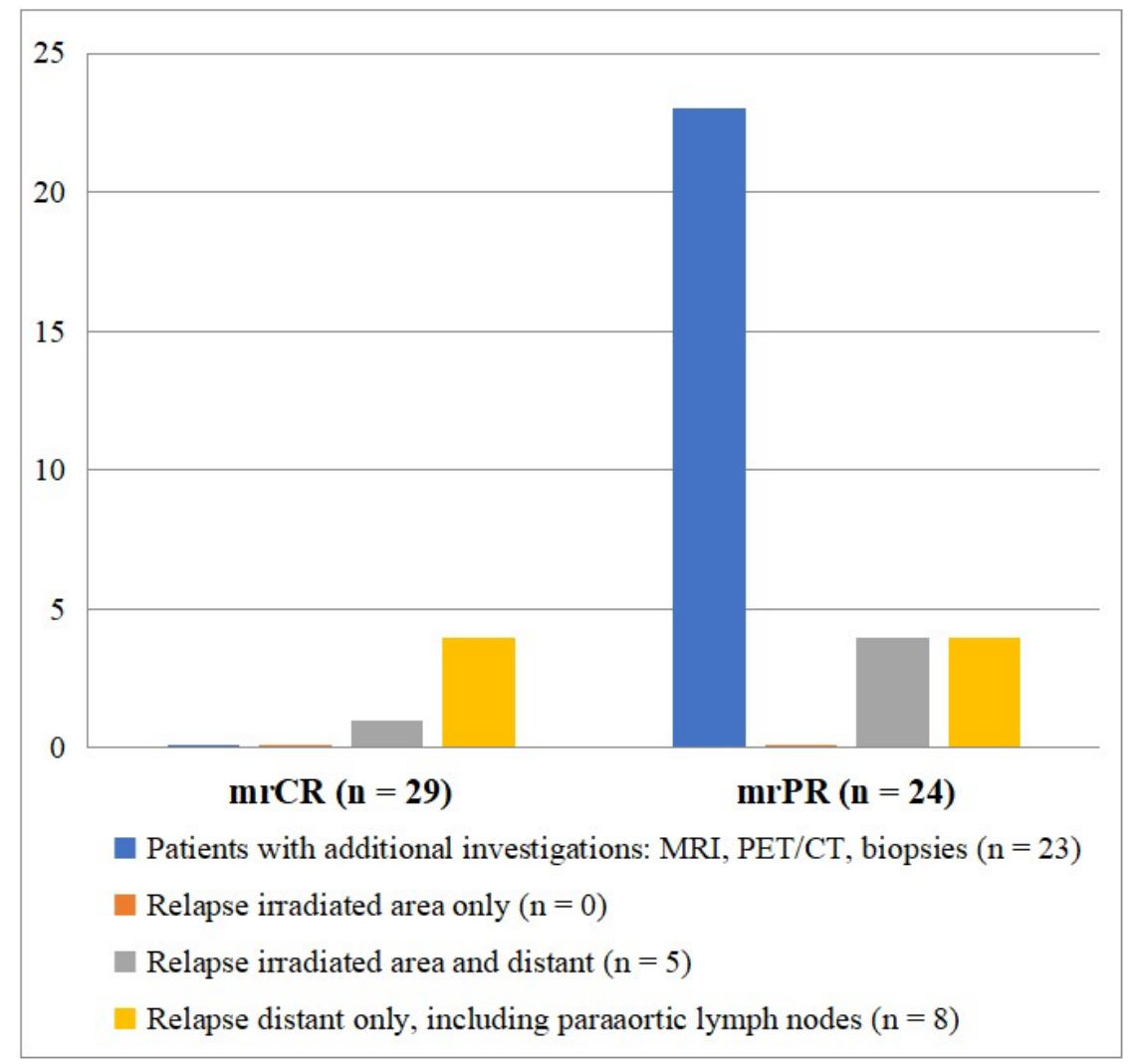

Figure 2. Additional investigations and relapse in $\mathrm{mrCR}$ and $\mathrm{mrPR}$.

Of the 13 patients who had a relapse, 2 (15\%) were alive and disease-free on follow-up. One patient received radiotherapy due to a para-aortic lymph node metastasis and the other received chemotherapy due to lung metastasis, 22 and 5 months from FUMR3m, respectively.

There were no statistically significant differences in overall survival (OS) between the groups with mrCR and $\mathrm{mrPR}$ at $\mathrm{FU} \mathrm{MR} 3 \mathrm{~m}(\mathrm{HR}=2.2, P=0.21)$. However, patients with $\mathrm{mrPD}$ at FU MR3m had significantly worse OS with a median survival time of nine months (range 1-40 months, $\mathrm{HR}=30, P<0.0001$ ) [Figure 3]. Three-year absolute survival rate was $85 \%, 89 \%, 78 \%$ and $14 \%$ for the whole group, $\mathrm{mrCR}, \mathrm{mrPR}$ and $\mathrm{mrPD}$, respectively. Five-year absolute survival rate was $81 \%, 85 \%, 74 \%$ and $0 \%$, respectively. There was no difference in overall survival OS after recurrence between the two groups (mrCR and $\mathrm{mrPR}$ ). 


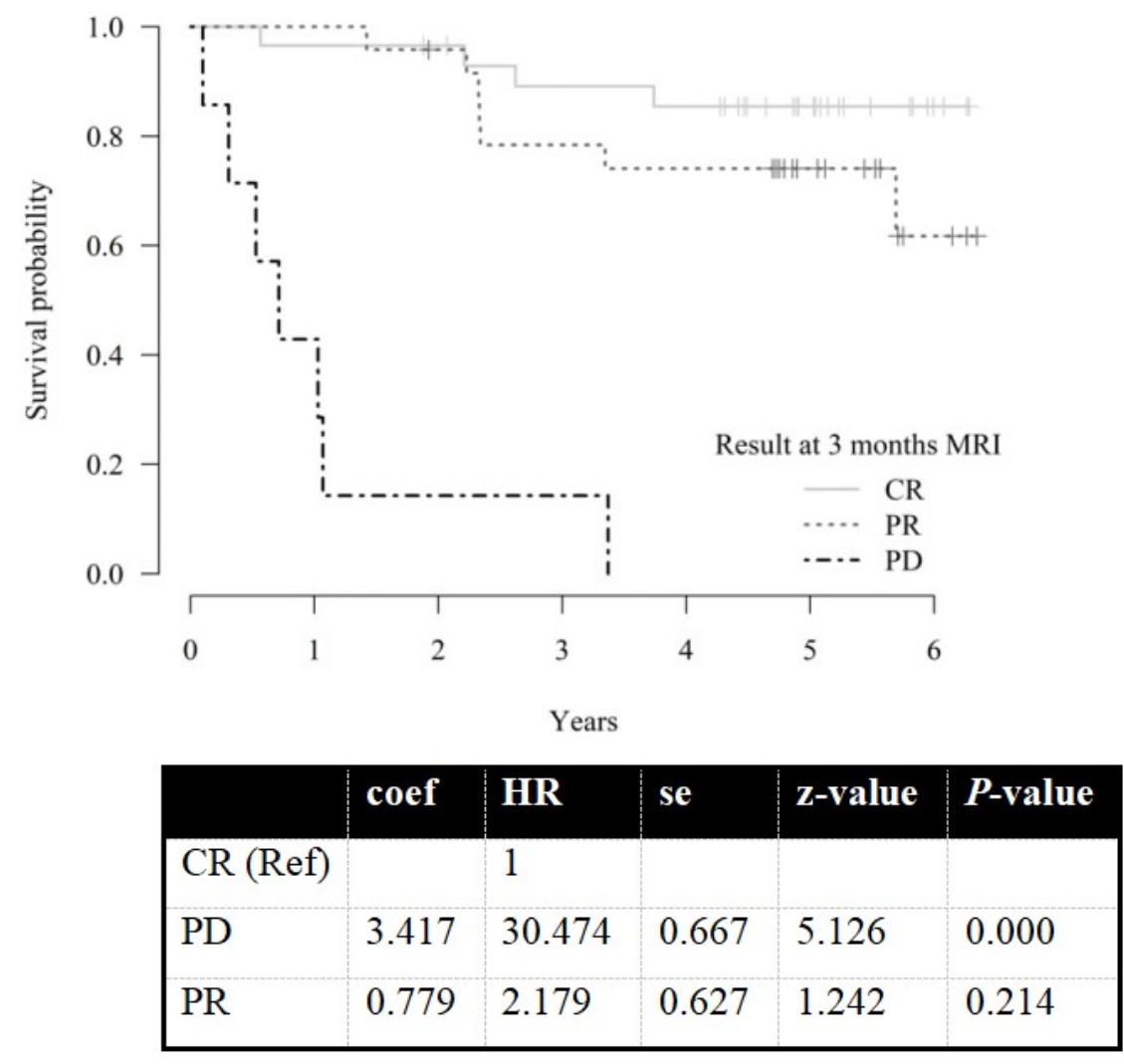

Figure 3. Overall survival illustrated using Kaplan-Meier curves.

\section{DISCUSSION}

This retrospective study was performed to assess the impact of FU MR3m after CRT of CC on further follow-up interventions and outcome regarding relapse and survival. There is sparse literature investigating this issue.

Due to suspicious remaining cervical tumour in $38 \%$ of the patients, several additional procedures were performed up to one year after the end of treatment. Thus, FU MR3m in this setting had a high impact on further clinical management, although none of these procedures resulted in findings of residual disease, i.e., vital tumour tissue. In more than half of the patients with $\mathrm{mrPR}$, the appearance of the post-treatment changes remained on repeated MRI examinations, although not consisting of residual tumour. The locoregional control rate for the patients with mrPR on FU MR3m and for the entire study population was very high ( $96 \%$ and $92 \%$, respectively), which is similar to what has been reported previously ${ }^{[20]}$.

Only two patients had cervical relapse, combined with distant metastasis. Similar to previous studies, one quarter of the patients in our study relapsed, but none in the group with mrCR had cervical relapse ${ }^{[21,22]}$. There was a higher rate of recurrences in mrPR compared to mrCR at FU MR3m, but the difference was not statistically significant. Interestingly, most patients with mrCR recurred with distant metastasis alone, compared to patients with $\mathrm{mrPR}$ where $38 \%$ had a combination of locoregional and distant recurrences. Patients with mrCR also recurred earlier than those with mrPR. However, there was no significant difference in overall survival between mrCR and mrPR; only patients with mrPD on FU MR3m had a significantly worse survival. This indicates that the result on FU MR3m had low impact regarding relapse and survival in patients with mrCR and mrPR. 
Kim et al. ${ }^{[1]}$ reported the disease course in 53 patients with stage IB1-IVA with residual disease using MRI three months after CRT for locally advanced CC. They found that $60 \%$ of the patients with residual tumour at three-month follow-up did not show further progression without any treatment and concluded that careful observation with close follow-up might be feasible in selected patients, especially those with a residual tumour size $\leq 2 \mathrm{~cm}^{[11]}$.

In our study, some patients had residual changes measuring up to $5 \mathrm{~cm}$ and yet did not later have cervical relapse. We also found that patients with mrPR significantly more often had advanced FIGO stage than the mrCR group and that the mrPR group had larger tumours than the mrCR group, but the difference was not significant. This might indicate that, in patients with more advanced stage and larger tumours, it might take a longer time to reach radiological complete remission in the cervix.

To our knowledge, the present study is the only one in the literature, besides the one by Kim et al.$^{[11]}$, which has reported on the course and management of suspected residual disease on MRI in a material where all patients have been selected to careful observation and not salvage hysterectomy.

The strength of this study is that it is a thorough and long follow-up of a consecutive and population-based (registry-based) patient material in an area where there is a lack of knowledge on the appropriate posttreatment follow-up with MRI.

There are several limitations with this study. First, it is a retrospective study including only a relatively small number of patients. The inclusion period is not recent, and there has been a change in the latest Swedish National Guidelines for CC, where the recommendation for pre-treatment evaluation now is MRI and $\mathrm{PET} / \mathrm{CT}$ and for post-treatment evaluation MRI or PET/CT 3-6 months after treatment.

In clinical practice, the MR examinations were reviewed by several radiologists. In the study, no MR images were reviewed; only the radiology reports and medical records were reviewed. Not all patients had the same MRI protocol since diffusion weighted sequences were added to the protocol in May 2012, about half-way through the study.

The optimal timing for evaluation of the therapeutic response to definitive CRT is controversial because the regression pattern of the tumour is variable due to heterogeneity of the cervical tumour and the responsiveness to radiotherapy. In addition, the optimal imaging modality for treatment control is controversial. Monitoring with repeated MRI, other imaging modalities and investigations are resource demanding for the healthcare system. In addition, the patients invested time to do the examinations, and associated anxiety waiting for the results must also be considered.

MRI three months after treatment is probably too early to evaluate locoregional response; however, many of the patients with remaining lesions in the cervix still had signal changes far after six months, and in some cases the signal changes never disappeared during follow-up. In fact, current treatment results in high locoregional control with a low frequency of relapses in previously irradiated areas. Better methods are needed for more accurate characterisation of remaining signal changes on MRI. Since many patients had residual changes without significance on repeated follow-up MRI and recurrence outside the irradiated area is of greater concern, PET/CT may be a better modality for treatment evaluation, although its availability must be considered. 
The type of imaging and timing of post-treatment evaluation need to be further studied, but it probably should be more individually planned in the future depending on tumour characteristics, risk of recurrence and treatment response during chemoradiotherapy. The main challenge in this patient group is prevention and discovery of distant relapses and to find out what characterises the patients who never reach locoregional control and/or develop distant metastases.

In conclusion, if residual changes are found on treatment response evaluation with MRI of the irradiated area at three months after the end of CRT and regarded as remaining tumour, this may lead to unnecessary additional investigations without impact on patient outcome. Instead, a remaining lesion with uniform low signal intensity on $\mathrm{T} 2$-weighted images should possibly be recognised as a potential complete response. In addition, absent contrast enhancement and no restricted diffusion may support this assumption but needs to be further evaluated in future studies.

Whether other or additional imaging modalities should be considered for the most appropriate treatment evaluation and avoidance of unnecessary investigations must be further investigated. However, welldesigned clinical trials also including PET/CT and multiparametric MRI (or PET/MRI) aimed at patients with residual disease resulting in appropriate response criteria are needed to elucidate the most appropriate management, careful observation or intervention, optimal timing and method of imaging and for early detection of recurrences outside the irradiated area, which might increase survival for patients with uni- or oligofocal metastasis.

\section{DECLARATIONS}

\section{Acknowledgments}

The authors express their thanks to Drs. Magnus Tengvar, Susanne Fridsten, Fredrik Strand and Anna Kistner, to radiology nurse Roberto Vargas, and to statistician Eva Hagel and Roger Halldin, for their comments and help with the preparation of this study. We also would like to thank the Swedish Quality Registry of Gynaecologic Cancer (SQRGC) and the Swedish Gynaecologic cancer group (SweGCG).

\section{Authors' contributions}

Made substantial contributions to conception and design of the study and performed data analysis and interpretation: Sundström H, Blomqvist L, Hellman K

Performed data acquisition, as well as provided administrative, technical, and material support: Sundström H, Blomqvist L, Hellman K

\section{Availability of data and materials}

The data that support the findings of this study are available from the corresponding author, HS, upon reasonable request.

\section{Financial support and sponsorship}

None.

\section{Conflicts of interest}

All authors declared that there are no conflicts of interest.

\section{Ethical approval and consent to participate}

The study was accepted by the regional ethical review board (Dnr 2017/1787-31) who deemed separate study-specific consent as not needed. 


\section{Consent for publication}

The study was accepted by the regional ethical review board (Dnr 2017/1787-31) who deemed separate study-specific consent as not needed.

\section{Copyright}

(c) The Author(s) 2021.

\section{REFERENCES}

1. WHO Estimated number of new cases of uterine cervical cancer in 2020 worldwide. Available from: https:/gco.iarc.fr/today/data/factsheets/cancers/23-Cervix-uteri-fact-sheet.pdf [Last accessed on 10 Aug 2021 ].

2. Cancer incidence in Sweden. Available from: https://www.socialstyrelsen.se/statistik-och-data/register/alla-register/cancerregistret/ [Last accessed on 10 Aug 2021].

3. for Cervical Cancer Meta-analysis Collaboration (CCCMAC). Reducing uncertainties about the effects of chemoradiotherapy for cervical cancer: individual patient data meta-analysis. Cochrane Database Syst Rev 2010;2010:CD008285. DOI PubMed PMC

4. Castelnau-Marchand P, Chargari C, Maroun P, et al. Clinical outcomes of definitive chemoradiation followed by intracavitary pulseddose rate image-guided adaptive brachytherapy in locally advanced cervical cancer. Gynecol Oncol 2015;139:288-94. DOI PubMed

5. Eifel PJ, Winter K, Morris M, et al. Pelvic irradiation with concurrent chemotherapy versus pelvic and para-aortic irradiation for highrisk cervical cancer: an update of radiation therapy oncology group trial (RTOG) 90-01. J Clin Oncol 2004;22:872-80. DOI PubMed

6. Gaffney DK. Optimal therapy for IB2 and IIA2 cervical cancer: surgery or chemoradiotherapy? J Gynecol Oncol 2012;23:207-9. DOI PubMed PMC

7. Green JA, Kirwan JM, Tierney JF, et al. Survival and recurrence after concomitant chemotherapy and radiotherapy for cancer of the uterine cervix: a systematic review and meta-analysis. Lancet 2001;358:781-6. DOI PubMed

8. Pecorelli S. Revised FIGO staging for carcinoma of the vulva, cervix, and endometrium. Int J Gynaecol Obstet 2009;105:103-4. DOI PubMed

9. Bhatla N, Berek JS, Cuello Fredes M, et al. Revised FIGO staging for carcinoma of the cervix uteri. Int J Gynaecol Obstet 2019;145:129-35. DOI PubMed

10. Elit L, Fyles AW, Devries MC, Oliver TK, Fung-Kee-Fung M; Gynecology Cancer Disease Site Group. Follow-up for women after treatment for cervical cancer: a systematic review. Gynecol Oncol 2009;114:528-35. DOI PubMed

11. Kim JY, Byun SJ, Kim YS, Nam JH. Disease courses in patients with residual tumor following concurrent chemoradiotherapy for locally advanced cervical cancer. Gynecol Oncol 2017;144:34-9. DOI PubMed

12. Shim SH, Kim SN, Chae SH, Kim JE, Lee SJ. Impact of adjuvant hysterectomy on prognosis in patients with locally advanced cervical cancer treated with concurrent chemoradiotherapy: a meta-analysis. J Gynecol Oncol 2018;29:e25. DOI PubMed PMC

13. Bipat S, Glas AS, Velden JVD, Zwinderman AH, Bossuyt PM, Stoker J. Computed tomography and magnetic resonance imaging in staging of uterine cervical carcinoma: a systematic review. Gynecologic Oncology 2003;91:59-66. DOI PubMed

14. Rockall AG, Ghosh S, Alexander-Sefre F, et al. Can MRI rule out bladder and rectal invasion in cervical cancer to help select patients for limited EUA? Gynecol Oncol 2006;101:244-9. DOI PubMed

15. Sahdev A, Sohaib SA, Wenaden AE, Shepherd JH, Resnek RH. The performance of magnetic resonance imaging in early cervical carcinoma: a long-term experience. Int J Gynecol Cancer 2007;17:629-36. DOI PubMed

16. Manganaro L, Lakhman Y, Bharwani N, et al. Staging, recurrence and follow-up of uterine cervical cancer using MRI: updated guidelines of the European Society of Urogenital Radiology after revised FIGO staging 2018. Eur Radiol 2021. DOI PubMed

17. Schreuder SM, Lensing R, Stoker J, Bipat S. Monitoring treatment response in patients undergoing chemoradiotherapy for locally advanced uterine cervical cancer by additional diffusion-weighted imaging: a systematic review. J Magn Reson Imaging 2015;42:57294. DOI PubMed

18. Cibula D, Pötter R, Planchamp F, et al. The European Society of Gynaecological Oncology/European Society for Radiotherapy and Oncology/European Society of Pathology guidelines for the management of patients with cervical cancer. Radiother Oncol 2018;127:404-16. DOI PubMed

19. Gotland RCCS. Regional guidelines for cervical, vaginal and vulvar cancer 2008. Avaliable from: https://www.yumpu.com/sv/document/read/23772070/regionalt-vardprogram-for-cervix-vaginal-och-vulvacancer-2008 [Last accessed on 9 Nov 2021].

20. Pötter R, Tanderup K, Schmid MP, et al. MRI-guided adaptive brachytherapy in locally advanced cervical cancer (EMBRACE-I): a multicentre prospective cohort study. Lancet Oncol 2021;22:538-47. DOI PubMed

21. Ferrandina G, Margariti PA, Smaniotto D, et al. Long-term analysis of clinical outcome and complications in locally advanced cervical cancer patients administered concomitant chemoradiation followed by radical surgery. Gynecol Oncol 2010;119:404-10. DOI PubMed

22. Touboul C, Uzan C, Mauguen A, et al. [Survival and prognostic factors after completion surgery in patients undergoing initial chemoradiation therapy for locally advanced cervical cancer]. Gynecol Obstet Fertil 2011;39:274-80. DOI PubMed 